\title{
The effect of metabolic syndrome on heart rate turbulence in non-diabetic patients
}

\author{
Alim Erdem ${ }^{1}$, Masahiro Uenishi ${ }^{2}$, Zekeriya Küçükdurmaz ${ }^{2}$, Kazuo Matsumoto², \\ Ritsushi Kato ${ }^{2}$, Motoki Hara ${ }^{2}$, Mehmet Yazici ${ }^{1}$ \\ ${ }^{1}$ Department of Cardiology, Abant Izzet Baysal University, Medical Faculty, Bolu, Turkey \\ ${ }^{2}$ Department of Cardiology, Saitama Medical University, Arrhythmia Research Center, Saitama, Japan
}

\begin{abstract}
Background: Metabolic syndrome (MetS), which includes a cluster of risk factors, is being increasingly recognized as a new risk factor for cardiovascular disease. Heart rate turbulence (HRT) is a Holter-based non-invasive method for detecting cardiac autonomic imbalance and is an independent, powerful predictor of cardiac arrhythmias and sudden cardiac death in different patient groups. This study evaluated the effect of MetS on HRT in non-diabetic patients.
\end{abstract}

Methods: This study included 80 non-diabetic MetS subjects and 50 healthy subjects. All 130 subjects underwent a 24-h ambulatory Holter electrocardiogram recording. Two indices of HRT were analyzed: turbulence onset (TO) and turbulence slope (TS). HRT values were classified into 3 categories for risk stratification: 1) Category 0, TO and TS were normal; 2) Category 1, either TO or TS was abnormal; 3) Category 2, both TO and TS were abnormal. Results: When we compared MetS rates in the HRT risk stratification groups, there were significant differences for all groups as compared with the controls (Category $0=$ MetS 28.8\%, $n=15$, Control 71.2\%, $n=37, p<0.001$; Category $1=$ MetS 80.8\%, $n=42$, Control $19.2 \%, n=10, p<0.001$; Category $2=$ MetS 88.5\%, $n=23$, Control 11.5\%, $n=3, p<0.001$ ). In addition, TO and TS abnormalities were correlated with the number of MetS components $(r=0.608, p<0.001 ; r=-0.388, p<0.001$, respectively).

Conclusions: To our knowledge, this is the first study to establish a relationship between $H R T$ and MetS. These findings suggest that MetS adversely affects HRT scores. In addition, the number of MetS components is related to impaired HRT scores. (Cardiol J 2012; 19, 5: 507-512)

Key words: metabolic syndrome, heart rate turbulence, cardiovascular risk

\section{Introduction}

Heart rate turbulence (HRT) is a Holter-based non-invasive method for detecting cardiac autonomic imbalance. This evaluation method is based on the physiological sinus node response to a premature ventricular beat [1]. Cardiac autonomic im- balance, manifested by impaired HRT, is a powerful risk factor for sudden cardiac death and malignant ventricular arrhythmia in patients with myocardial infarction or congestive heart failure $[2,3]$.

Metabolic syndrome (MetS) consists of a group of cardiovascular (CV) risk factors that includes abdominal obesity, dyslipidemia, hypertension, and

Address for correspondence: Alim Erdem, MD, Department of Cardiology, Abant Izzet Baysal University, Medical Faculty, Bolu, Turkey, tel: +905317741266, fax: +903742534615, e-mail: cardiology14@gmail.com 
impaired glucose metabolism. Despite the ties to dyslipidemia, insulin resistance, elevated blood pressure, and abdominal obesity, the main pathophysiological mechanism underlying the increased $\mathrm{CV}$ risk is unclear. Autonomic dysfunction has also been implicated in the pathophysiology of MetS [4]. MetS is associated with a 2 -fold increase in CV outcomes and a 1.5-fold increase in all-cause mortality [5]. Cardiac autonomic dysfunction may contribute to the pathophysiological process of MetS with respect to CV outcomes. We hypothesized that the increased CV risk of MetS was related to cardiac autonomic dysfunction.

This study evaluated the relationship between MetS and cardiac autonomic dysfunction as detected by HRT. Since diabetic neuropathy and autonomic dysfunction in diabetic patients may affect HRT analysis, the study evaluated non-diabetic patients.

\section{Methods}

\section{Patients}

Eighty non-diabetic patients with MetS and 50 healthy age and sex matched subjects from Saitama Medical University Hospital Cardiology Department outpatient clinics were enrolled in this study. The control group consisted of 50 healthy persons, whose Holter recordings demonstrated ventricular premature beats (VPB) suitable ( $>6 /$ day) for HRT analysis. Subjects were excluded if they had rhythms other than sinus as atrial fibrillation or flutter, diabetes mellitus, coronary artery disease, an implanted permanent pacemaker, drug use that may influence HRT such as; beta blockers, $\mathrm{Ca}++$ channel blockers, Group I and IV antiarrhythmics, ACE inhibitors, etc., inadequate VPB to measure HRT, and refusal to provide informed consent for the study. The study was approved by the local ethic committee and all subjects gave written informed consent.

\section{Anthropometric analysis}

Body weight and height was measured with the patient in light clothes without shoes to the nearest $0.5 \mathrm{~kg}$ and $0.5 \mathrm{~cm}$. Waist circumference was measured horizontally in quiet expiration, halfway between the lower rib and the iliac crest. Body mass index was calculated as weight $[\mathrm{kg}]$ divided by height squared [m].

\section{Metabolic syndrome definition}

The presence of MetS was established according to National cholesterol education program-adult treatment panel III criteria, using the Asian Pacific
World Health Organization modification [1]. MetS was diagnosed if more than 3 of the following components are present; a) abdominal obesity: waist circumference $>90 \mathrm{~cm}$ in men and $>80 \mathrm{~cm}$ in women; b) triglyceride level $1.7 \mathrm{mmol} / \mathrm{L}$ or greater; c) HDL-cholesterol less than or equal to $1.0 \mathrm{mmol} / \mathrm{L}$ in men and less than or equal to $1.3 \mathrm{mmol} / \mathrm{L}$ in women; d) blood pressure: $\geq 130 / 85 \mathrm{~mm} \mathrm{Hg}$; e) fasting glucose level of $6.1 \mathrm{mmol} / \mathrm{L}$ or greater.

\section{Holter monitoring and HRT analysis}

The 24-h Holter recordings of all patients were analyzed to obtain the HRT parameters of turbulence onset (TO) and turbulence slope (TS). Recordings were performed with a GE Marquette SEER system digitizing at 125 samples per second (GE Marquette, Milwaukee, WI). QRS detection, morphology classification (normal, aberrant, premature aberrant) and measurement of the RR interval were automatically performed by the system. All Holter files were reviewed and manually corrected. HRT analysis was performed on sequences of sinus RR intervals after VPB. The evaluated sinus rhythm immediately before and after the VPB was free from any arrhythmia or other artifacts. The HRT after a VPB comprises two parameters: TO, which represents the initial acceleration (shortening of R-R intervals); and TS, which represents the subsequent deceleration (prolongation of R-R intervals) [2]. In mathematical terms, TO (\%) (normal $<0)$ is the difference between the sum of the first two R-R intervals after the compensatory pause following a VPB and the sum of the last two R-R intervals preceding the $\mathrm{VPB}$, divided by the sum of the last two R-R intervals preceding the VPB. The TS (normal $>2.5 \mathrm{~ms} / \mathrm{R}-\mathrm{R}$ interval number) were accepted as the steepest slope of a regression line over any sequence of five consecutive R-R intervals. The average of HRT values measured for all convenient VPBs was accepted as the final HRT value to characterize the patient [3, 4]. For the risk stratification HRT values [5] are classified into 3 categories: 1) Category 0: TO and TS are normal; 2) Category 1: one of TO or TS is abnormal; 3) Category 2: both TO and TS are abnormal.

\section{Statistical analysis}

All statistical analyses were performed using the SPSS software package 15.0 (SPSS Inc, Chicago, IL, USA). Data are presented as frequencies and percentages for categorical variables and mean $\pm \mathrm{SD}$ for continuous variables. Differences between 2 groups were assessed using the $\chi^{2}$ and unpaired t-tests. Because total onset, total slope and MetS 
Table 1. Baseline characteristics of the two study groups.

\begin{tabular}{lccc}
\hline & MetS group $(\mathbf{n}=\mathbf{8 0})$ & Control group $(\mathbf{n}=\mathbf{5 0})$ & $\mathbf{P}$ \\
\hline Age [years] & $42.1 \pm 5.0$ & $41.0 \pm 5.1$ & 0.351 \\
Gender (male/female) & $38 / 42$ & $24 / 26$ & 0.647 \\
Body mass index [kg/m $]$ & $28.7 \pm 4.6$ & $28.3 \pm 4.9$ & 0.693 \\
Waist circumference [cm]: & & $94.8 \pm 11.7$ & 0.041 \\
$\quad$ Men & $103.1 \pm 16.8$ & $95.5 \pm 12.4$ & 0.032 \\
$\quad$ Women & $107.2 \pm 12.7$ & $94.3 \pm 11.4$ & 0.306 \\
Fasting plasma glucose [mg/dL] & $101.7 \pm 21.2$ & $105.0 \pm 44.2$ & 0.028 \\
Triglyceride [mg/dL] & $161.6 \pm 65.4$ & $46 \pm 12$ & 0.038 \\
HDL-cholesterol [mg/dL] & $41 \pm 8$ & $13(26.0 \%)$ & 0.012 \\
Current smoking & $42(52.5 \%)$ & $123.3 \pm 6.4$ & 0.009 \\
Systolic blood pressure [mm Hg] & $132.5 \pm 8.5$ & $72.4 \pm 6.2$ & 0.011 \\
Diastolic blood pressure [mm Hg] & $89.2 \pm 8.7$ & & \\
\hline
\end{tabular}

values were not normally distributed, between-group differences were assessed by the one-way ANOVA. Pearson's and Spearman's correlation coefficients were used to test the relationship between the TO, TS and MetS factors. Linear logistic regression analysis was performed to identify the independent determinants of MetS (including systolic blood pressure, diastolic blood pressure, waist circumference, triglyceride levels, HDL-cholesterol levels, fasting glucose, TO and TS). A value of $p<0.05$ was considered statistically significant.

\section{Results}

Eighty MetS patients (38 [47.5\%] male, 42 [52.5\%] female) and 50 healthy control (24 [48.0\%] male, $26[52.0 \%]$ female) were included in this study. MetS group clinical characteristics were shown in Table 1 . When we compare the MetS rates with respect to the HRT risk stratification groups, there were significant differences for all category (Category $0=$ MetS 28.8\%, n: 15, Control 71.2\%, n: 37, p < 0.001; Category $1=$ MetS 80.8\%, n: 42, Control 19.2\%, n: 10, p < 0.001; Category $2=$ MetS $88.5 \%$, n: 23, Control 11.5\%, n: 3, p < 0.001, respectively) (Fig. 1). TO and TS values of MetS group were significantly different compared with

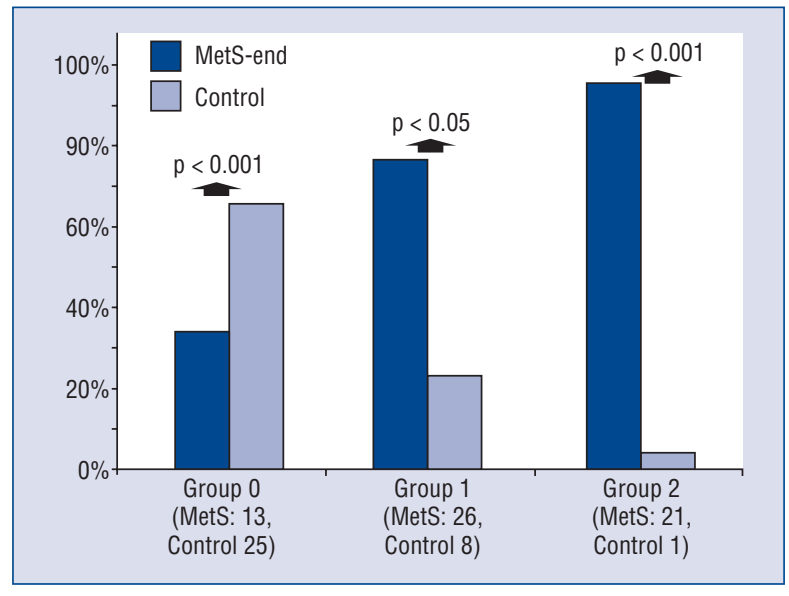

Figure 1. Metabolic syndrome (MetS) rates with respect to the heart rate turbulence risk stratification group.

the control group $(0.36 \pm 1.40$ vs. $-0.49 \pm 1.03$, $\mathrm{p}<0.001 ; 2.44 \pm 1.37$ vs. $1.92 \pm 0.70, \mathrm{p}=0.012$, respectively).

In the subgroup analysis of MetS group, patients were compared in number of MetS components, there were statistically significant difference in TO and TS values (Table 2). Also a strong positive correlation between the number of MetS com-

Table 2. Subgroup analysis of metabolic syndrome (MetS) group in total onset and total slope.

\begin{tabular}{lcccc}
\hline & MetS (mc: 3) & MetS (mc: 4) & MetS (mc: 5) & P \\
\hline Total onset & $-0.5 \pm 1.2$ & $0.7 \pm 0.9$ & $1.6 \pm 1.1$ & $<0.001$ \\
Total slope & $3.2 \pm 0.5$ & $2.0 \pm 1.3$ & $1.4 \pm 1.2$ & $<0.001$ \\
\hline
\end{tabular}

$\mathrm{mc}-$ number of the metabolic syndrome components 


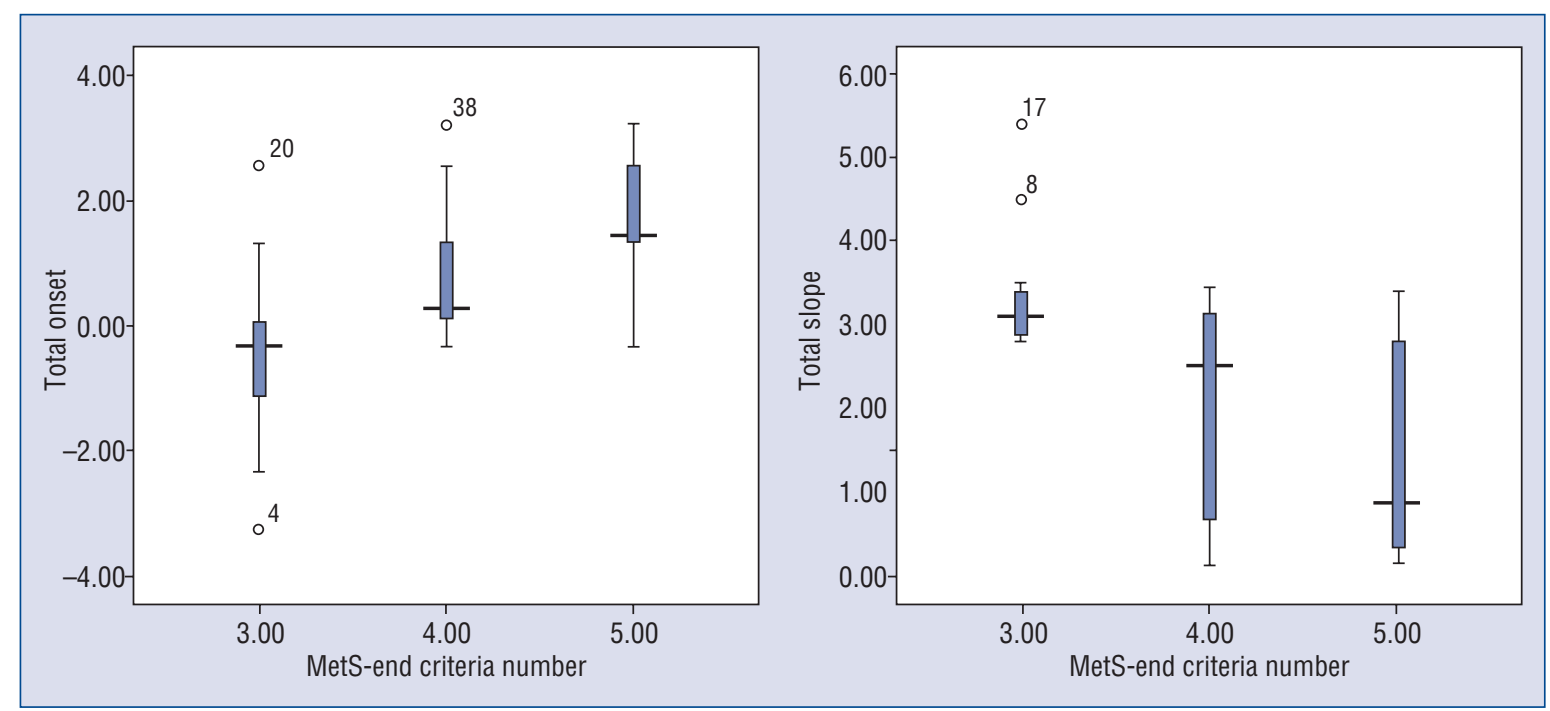

Figure 2. Correlation between metabolic syndrome (MetS) criteria number and heart rate turbulence parameters.

ponents and TO, a moderate negative correlation between the number of MetS components and TS were detected $(\mathrm{r}=0.608, \mathrm{p}<0.001, \mathrm{r}=-0.388$, $\mathrm{p}<0.001$ ) (Fig. 2). In linear logistic regression analysis (including systolic blood pressure, diastolic blood pressure, waist circumference, triglyceride levels, HDL-cholesterol, fasting glucose, TO and TS) waist circumference, systolic blood pressure, triglyceride levels and TO was found to be significant and independent predictors of MetS (Table 3).

Patients who have 5 components of MetS were more likely than the 3 components MetS patients to have Category 2 (adjusted odds ratio $[\mathrm{OR}]=1.4$; $95 \%$ confidence interval $[\mathrm{CI}]=1.03-2.03 ; \mathrm{p}=0.04$ ). Patients who have 4 criteria of MetS were not more likely than 3 criteria MetS group to have Category 1 turbulence (adjusted OR $=1.2 ; 95 \% \mathrm{CI}=0.8$ $-1.9 ; \mathrm{p}=0.29$ ) but were more likely to have Category 2 turbulence (adjusted OR $=1.8 ; 95 \% \mathrm{CI}=1.1-$ $-3.0 ; p=0.03$ ). All group of MetS were more likely than the Control group to have Category 1 or Category 2 turbulence (respectively, $2.3 ; 95 \% \mathrm{CI}=1.1$ $-4.9 ; \mathrm{p}=0.03 ; 1.4 ; 95 \% \mathrm{CI}=1.03-2.03 ; \mathrm{p}=0.04$; $1.3 ; 95 \% \mathrm{CI}=1.1-1.9 ; \mathrm{p}=0.038$ ).

A statistically significant difference between the two groups was found in terms of VPB number detected by 24-h Holter (MetS: $36.73 \pm 28.81$; Control: $10.58 \pm 10.31 ; \mathrm{p}<0.001)$. A statistically strong significant positive correlation was found between the number of VPB and number of MetS components $(r=0.695, p<0.001)$. When 3 groups according to the number of MetS components were formed, a statistically significant difference was
Table 3. Linear logistic regression analysis of variables associated with metabolic syndrome.

\begin{tabular}{lccc}
\hline & Beta & $95 \% \mathbf{C l}$ & $\mathbf{P}$ \\
\hline Fasting glucose & 0.285 & $0.001-0.002$ & 0.250 \\
Waist circumference & 0.372 & $0.006-0.014$ & $<0.001$ \\
Triglyceride & 0.290 & $0.001-0.002$ & 0.005 \\
HDL-cholesterol & 0.033 & $-0.010-0.007$ & 0.394 \\
Systolic BP & 0.321 & $0.004-0.018$ & 0.002 \\
Diastolic BP & 0.127 & $-0.003-0.014$ & 0.184 \\
Total onset & 0.201 & $0.002-0.012$ & 0.009 \\
Total slope & 0.031 & $-0.002-0.001$ & 0.588 \\
\hline
\end{tabular}

$\mathrm{Cl}$ - confidence interval; $\mathrm{BP}$ - blood pressure

found in terms of the VPB number among the groups $(12.85 \pm 4.59 ; 40.60 \pm 34.93 ; 80.34 \pm 67.80$; $\mathrm{p}<0.001$, respectively).

\section{Discussion}

The principal findings of our study were that 1) MetS patients were more likely to have abnormal HRT than the control group, 2) the TO and TS were significantly different between the MetS and Control groups, 3) there were significant differences in TO and TS in the MetS subgroup analysis when comparing the number of MetS components, 4) TO was a significant, independent predictor of MetS presence, and 5) there was a significant positive correlation between the numbers of VPBs and MetS components. 
The heart is highly innervated by vagal and sympathetic fibers and is very sensitive to autonomic influences [6]. The autonomic nervous system responds to all changes sensed by baroreceptors and chemoreceptors to maintain CV homeostasis [2]. Studies have shown that cardiac autonomic dysfunction plays an essential role in the development of CV disease in MetS patients [7, 8]. HRT is an important parameter for evaluating cardiac autonomic functions. A blunted HRT reflects cardiac autonomic dysfunction, especially reduced baroreflex sensitivity [5]. HRT is reduced not only following myocardial infarction, but also in patients with a variety of diseases affecting the heart, congestive heart failure, and possibly diabetes. Further, HRT may provide clinically useful risk stratification information [9-11].

Many studies have suggested that MetS is a powerful predictor of the risk of developing $\mathrm{CV}$ disease [12]. In a meta-analysis of 21 studies, CV disease, coronary heart disease, stroke, and mortality were more prevalent in patients with MetS than in patients without it [13]. We found a similar result in that MetS patients demonstrated abnormal HRT more often than the control group. In addition, the MetS group showed abnormal TO and TS values as compared with the control group. We believe that the relationship between MetS and CV disease development is related to cardiac autonomic dysfunction.

The number of MetS components is associated with CV mortality and morbidity $[7,8]$. Protorov and Glukhovsky [14] showed a significant correlation between arrhythmias and the number of MetS components. Similarly, we found that the number of MetS components was positively correlated with TO and negatively correlated with TS. Therefore, the number of MetS components, including abdominal obesity, low HDL-cholesterol levels, hypertriglyceridemia, high blood pressure, and high fasting glucose, is an important factor for increased CV risk.

Cardiac autonomic dysfunction in patients with MetS can be evaluated using different parameters, such as heart rate variability (and heart rate recovery. However, HRT has not been used in this situation. Studies have established a relationship between cardiac autonomic dysfunction and MetS. One study revealed that heart rate recovery is significantly impaired in young adult males with MetS [7]. Stein et al. [15] found that reduced heart rate variability was a valuable marker of cardiac autonomic dysfunction, and was associated with MetS, independent of fasting glucose levels. We also determined that HRT was impaired in MetS patients without diabetes mellitus as compared with healthy subjects. To our knowledge, this is the first study establishing a relationship between HRT and MetS.

Another important risk factor for CV mortality is VPBs [16]. Premature ventricular contractions leading to ventricular tachycardia, which can in turn degenerate into ventricular fibrillation, is a common mechanism underlying sudden cardiac death. Clinically, a decrease in premature ventricular contractions would improve ventricular function and sudden cardiac death [17]. Provotorov and Glukhovsky [18] found that patients with MetS more frequently had polymorphic VPBs associated with an elevated mean heart rate. Similarly, we found a significant difference between the MetS and Control groups in terms of VPBs and a significant positive correlation between the numbers of VPBs and MetS components.

\section{Limitations of the study}

The main limitation of our study was the small sample size. Since a small sample size results in low statistical power for equivalency testing, negative results may be simply due to chance. In addition, we did not examine the impact of circadian variation. Diurnal fluctuations in autonomic tone suggest one value for HRT in $24 \mathrm{~h}$. This may also influence the results.

\section{Conclusions}

In conclusion, our results revealed that HRT is significantly impaired in MetS patients without diabetes mellitus. HRT is a significant predictor of cardiac autonomic dysfunction and it is useful to identify MetS patients without diabetes mellitus who have potentially increased $\mathrm{CV}$ risk.

\section{Conflict of interest: none declared}

\section{References}

1. Executive Summary of The Third Report of The National Cholesterol Education Program (NCEP) Expert Panel on Detection, Evaluation, And Treatment of High Blood Cholesterol In Adults (Adult Treatment Panel III). JAMA, 2001; 285: 2486-2497.

2. Akselrod S, Gordon D, Ubel FA, Shannon DC, Berger AC, Cohen RJ. Power spectrum analysis of heart rate fluctuation: A quantitative probe of beat-to-beat cardiovascular control. Science, 1981; 213: 220-222.

3. Mrowka R, Persson PB, Theres H, Patzak A. Blunted arterial baroreflex causes ,pathological” heart rate turbulence. Regulatory, integrative and comparative physiology. Am J Physiology, 2000; 279: R1171-R1175.

4. Watanabe MA. Heart rate turbulence: A review. Indian Pacing Electrophysiol J, 2003; 3: 10-22. 
5. Bauer A, Malik M, Schmidt G et al. Heart rate turbulence: Standards of measurement, physiological interpretation, and clinical use: International Society for Holter and Noninvasive Electrophysiology Consensus. J Am Coll Cardiol, 2008; 52: 1353-1365.

6. Wang W, Ma R. Cardiac sympathetic afferent reflexes in heart failure. Heart Failure Rev, 2000; 5: 57-71.

7. Nesto RW. The relation of insulin resistance syndromes to risk of cardiovascular disease. Rev Cardiovasc Med, 2003; 4 (suppl. 6): S11-S18.

8. Mottillo S, Filion KB, Genest J et al. The metabolic syndrome and cardiovascular risk a systematic review and meta-analysis. J Am Coll Cardiol, 2010; 56: 1113-1132.

9. Schmidt G, Malik M, Barthel P et al. Heart-rate turbulence after ventricular premature beats as a predictor of mortality after acute myocardial infarction. Lancet, 1999; 353: 1390-1396.

10. Miwa Y, Miyakoshi M, Hoshida K et al. Heart rate turbulence can predict cardiac mortality following myocardial infarction in patients with diabetes mellitus. J Cardiovasc Electrophysiol, 2011; 22: 1135-1140.

11. Szymanowska K, Piatkowska A, Nowicka A et al. Clinical significance of heart rate turbulence assessment in patients with chronic heart failure. Kardiol Pol, 2008; 66: 1289-1295.
12. Kahn R, Buse J, Ferrannini E, Stern M. The metabolic syndrome: Time for a critical appraisal. Diabetes Care, 2005; 28: 2289.

13. Galassi A, Reynolds K, He J. Metabolic syndrome and risk of cardiovascular disease: A meta-analysis. Am J Med, 2006; 119: 812-819.

14. Provotorov VM, Glukhovsky ML. Rhythm and conductivity disorders in patients at the initial stages of metabolic syndrome. Klinicheskaia Meditsina, 2009; 87: 26-28.

15. Stein PK, Barzilay JI, Domitrovich PP et al. The relationship of heart rate and heart rate variability to non-diabetic fasting glucose levels and the metabolic syndrome: The Cardiovascular Health Study. Diabetic Medicine, 2007; 24: 855-863.

16. Beaufort-Krol G, Dijkstra SSP, Bink-Boelkens MTE. Natural history of ventricular premature contractions in children with a structurally normal heart: Does origin matter? Europace, 2008; 10: 998.

17. Sekiguchi Y, Aonuma K, Yamauchi $Y$ et al. Chronic hemodynamic effects after radiofrequency catheter ablation of frequent monomorphic ventricular premature beats. J Cardiovascular Electrophysiol, 2005; 16: 1057-1063.

18. Provotorov V, Glukhovsky M. Ventricular extrasystole in patients with metabolic syndrome. Kliniceskaia Medicina, 2010; 88: 29-31. 